\title{
Link between cancer stem cells and adult
} mesenchymal stromal cells: implications for

\section{cancer therapy}

\author{
"If MSCs are able to migrate to tumoral stroma, MSCs engineered to express \\ antitumor factors should be an innovative choice as a cell-mediated gene therapy \\ to counteract tumor growth."
}

\begin{abstract}
A new concept has emerged in tumor biology suggesting that tumoral growth is derived from cancer stem cells (CSCs) present in the tumor. Moreover, these CSCs share common features with adult stem cells. In parallel, recent works have highlighted interactions between multipotent stromal cells (MSCs) and carcinoma, and the possible use of MSCs in cell-based anticancer therapies. Thus, translational research between fields of stem cells and tumor biology has changed our perception of carcinoma progression. The therapeutic implications are considerable and imply that to eradicate cancer we need to identify and target the CSCs as well as the MSCs that have migrated to the stroma.
\end{abstract}

\section{Links between CSCs \& MSCs}

Adult stem cells are well defined and characterized by phenotype expression markers CD90, CD73 and CD105, by their ability to differentiate into lineage, including adipose tissue, chondrocyte or osteoblast, and their properties of asymmetric division with capacity for self renewal [1]. Adult stem cells are also highly immunosuppressive, and have been proposed as anti-inflammatory or immunosuppressive therapy in autoimmune diseases such as encephalitis or arthritis. MSCs interact on various lymphocyte populations such as NK lymphocytes, Th1 and Treg cells and cancer growth [2]. They act through the expression of prostaglandin nitric oxide and the indoleamine 2,3-dioxygenase enzyme responsible for decreasing the activity of tryptophan. Finally, they express many cytokines of the family of IL-6, especially after stimulation through IL-1 $\beta$.

MSCs express adhesion molecules that are mediators involved in the migration and homing of the cells to the bone marrow. They include the integrin family ( $\alpha 1 \beta 1$ and $\alpha 5 \beta 1$ ), the immunoglobulin superfamily (ICAM-1 and VCAM-1), CD44, the ligand of hyaluronic acid, and other molecules of the extracellular matrix (ECM) [3]. In addition, the stromal cells synthesize and assemble many molecules of the ECM, including fibronectins, laminins, collagens, tenascins and syndecans, and other glycosaminoglycans. Multipotent stromal cells express several pro-angiogenic factors including angiopoietin-1, VEGF, growth factors such as PDGF, FGF-2 and FGF-7, and cytokines (IL-6 and TNF- $\alpha$ ), interacting synergistically both on endothelial cells to promote vasculogenesis and on carcinoma cells $[4,5]$.

\section{"The therapeutic implications are considerable and imply that to eradicate cancer we need to identify and target the CSCs as well as the MSCs that have migrated to the stroma."}

Recent data show that the treatment of lung cancer by chemotherapy, including doxoribicin and cysplatin, causes resistent cells to express stem cell markers such as CD133, CD117, SSEA-3 and OCT 4 [6]. These cells retain a capacity for self-renewal that makes them very similar to CSCs. Once injected into immunocompromised mice, they will give rise to highly aggressive metastatic tumors. Tumorigenic prostate CSCs were isolated from human samples, and characterized by expression of CD44, $\alpha 2 \beta 1$ integrin phenotype and stem cells transcription factors (oct 4 , nanog and sox 2) [7]. They represented $0.1 \%$ of the tumor and did not correlate with metastatic stage of the disease. This suggests that these selected cells are stem cell-like progenitors. Three mechanisms may explain their emergence: the first is that cancer cells all derive from transformation of stem cells present in adult tissue; the second hypothesis is that only a restricted number of carcinoma cells acquire the stem cell

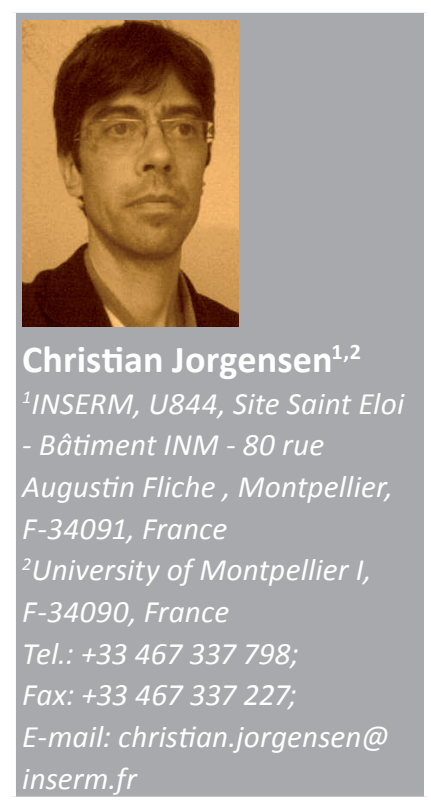

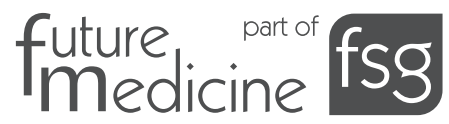


fate; and the last but nonexclusive mechanism is that the micro-environment of the tumor attracts stem cells to the stroma and promotes their transformation [8-11].

The comparative data of the CSCs and adult stromal cells show similarities. Thus, asymmetric division, the capacity for self-renewal, is shared by the two cell types. The expression of surface markers such as integrins, CD134, CD133 and CD49e are identical. Furthermore, the expression of transcription factors, such as OCT and $\beta$-atenin, and the involvement of the Hedgehog-GLI signaling pathway and notch and cytokines, such as IL-11 and CTGF, are shared by both CSCs and MSCs.

\section{MSCs \& stromal microenvironment}

The tumor stroma is composed of tumor-associated fibroblasts (TAFs), myeloid-derived suppressor cells, T cells and endothelial cells, all contributing to the microenvironment [14]. TAFs have been isolated and shown to stimulate transformation of prostatic epithelial cells through paracrine pathways [15]. The human fibroblastactivation protein- $\alpha$ is a $95 \mathrm{kD}$ cell-surface antigen selectively expressed in reactive stromal fibroblasts of epithelial cancers, granulation tissue of healing wounds, and malignant cells of bone and soft-tissue sarcomas. The properties in terms of expression of chemokine or differentiation capabilities of TAFs were never compared with adult stem cells, and the possibility of their presence in trapped mesenchymal stromal cells is not excluded.

Recent evidence also suggests that MSCs could be involved in the progression of carcinogenesis and could migrate towards primary tumors and metastatic sites [16]. In addition, it is thought that MSCs can modulate tumor growth, although this issue remains controversial and is not well understood. Depending on the origin of the tumor and number of MSCs, the results may lead to apoptosis of cancer cells or metastasis promotion. The antiproliferative action of MSCs was reported in a model of colon carcinogenesis in rats, in which the co-injection of MSCs with tumor cells in a gelatine matrix implanted subcutaneously led to growth inhibition [17]. Rat MSCs have the ability to migrate towards glioma cells and to inhibit their proliferation [18]. When implanted into the contralateral hemisphere, MSCs migrate to the hemisphere bearing the tumor. In an experimental model of Kaposi sarcoma, primary human MSCs exerted a potent tumor-suppressive effect in vivo through direct cell contact [19]. The in vivo tumor-suppressive effects of MSCs correlated with their ability to inhibit Akt activity. These findings suggest that human malignancies characterized by a dysregulated Akt may be specific targets of the antitumorigenic properties of MSCs.

By contrast, the immunosuppressive and pro-angiogenic properties of MSCs accounts, at least in part, for their effects on cancer development, and attest for the complexity of the dialog between MSCs and cancer cells. Loss of TGFRII in mammary carcinoma models led to increased numbers of pulmonary metastases associated with enhanced expression of CXCL1 and -5 , and ptg2 [20]. Moreover, the expression of chemokines such as CCL 5 by mesenchymal cells has been shown to promote the emergence of pulmonary metastases, showing a strong interaction between mammary cancer cells and adult mesenchymal cells [21]. Moreover, MSCs express chemokines such as IL-8/CXCL8, which are involved in the recruitment of endothelial progenitors [22]. Conversely, MSCs may recruit carcinoma cells circulating through SDF1/CXCR4 expressed by both MSCs and tumoral cells [23]. Thus, mesenchymal cell knockdown for both SDF1 and CXCR4 decreased the migration of tumor cells. SDF1/CXCR4 are involved in the recruitment of both carcinoma cells and mesenchymal stem cells through TAC1 expression.

\section{"A new concept has emerged in tumor biology suggesting that tumoral growth is derived from cancer stem cells ... present in the tumor."}

One of the characteristics of CSCs in the colon is the expression of both IL- 4 and its corresponding receptor. This cytokine is a survival factor for CD133+ cells. Thus, IL-4 inhibition prevented apoptosis through suppression of c-flip and $\mathrm{Bcl}-\mathrm{Xl}$ [24]. It is likely that the selection by chemotherapy favors selection of CSCs expressing a high concentration of IL-4.

The precise molecular interactions between mesenchymal cells, endothelial cells, CSCs and tumor cells remain to be determined in the development of metastasis. Other cells such as osteoblasts and osteoclasts contribute to the microenvironment. In multiple myeloma malignancy (MM), the formation of osteolytic lesions in the bone is enhanced by the interaction of MM with MSCs [25]. MMs secrete the Wnt inhibitor Dickkopf (Dkk)-1, which in turn prevents MSCs from differentiating into osteoblasts and supports plasmocyte growth through 
IL-6. Further studies are warranted to identify the soluble factors expressed by MSCs that support carcinoma growth.

\section{MSCs as a Trojan horse}

If MSCs are able to migrate to tumoral stroma, MSCs engineered to express antitumor factors should be an innovative choice as a cell-mediated gene therapy to counteract tumor growth [26]. For example, engineered MSCs were modified to express IFN- $\gamma$ and were able to inhibit the growth of melanoma and leukemia cells in vitro and in vivo [27]. Furthermore, MSCs infected with an adenovirus or retrovirus encoding IL-12 and injected intraperitoneally to mice 1 week before subcutaneous injection of melanoma cells, hepatoma cells or lung cancer cells are potent inhibitors of tumor growth [28]. The observed antitumoral effect of MSC-IL-12 was primarily mediated by activation of $\mathrm{NK}$ cells and $\mathrm{CD} 8^{+}$ $\mathrm{T}$ cells in the inhibition of metastasis formation and primary tumor growth. CX3CL1-expressing MSCs injected intravenously into syngeneic immunocompetent recipient-bearing lung metastases of C26 colon carcinoma or B16 melanoma cells were shown to target tumoral but not normal tissue, inducing both innate and adaptive anticancer immunity response and thus prolonging the animals' survival [29]. Mouse MSCs adenovirally transduced to express human NK4, an antagonist of HGF, exert potent antitumorigenic effects by inhibiting tumor-associated angiogenesis and lymphoangiogenesis and by inducing tumor cell apoptosis [30]. More recently, we showed that MSCs expressing a plasminogen inhibitor efficiently prevented growth and osteolysis of human prostatic cells lines in vivo [31].

These new data on the immunological properties of mesenchymal cells, the interactions between carcinoma and TAFs and the link between CSCs and MSCs justify the need for a first meeting dedicated to stem cells and cancer, which is to be held in Montpellier, France, in May 2009 [101]. These exciting contributions are possible through the emergence of the field of adult stem cells and CSCs, which may bring about new concepts and therapies for cancer.

\section{Financial \& competing interests disclosure}

The author has no relevant affliations or financial involvement with any organization or entity with a financial interest in or financial conflict with the subject matter or materials discussed in the manuscript. This includes employment, consultancies, honoraria, stock ownership or options, expert testimony, grants or patents received or pending, or royalties.

No writing assistance was utilized in the production of this manuscript.

\section{Bibliography}

1 Dominici M, Le Blanc K, Mueller I et al: Minimal criteria for defining multipotent mesenchymal stromal cells. The International Society for Cellular Therapy position statement. Cytotherapy 8, 315-317 (2006).

2 Djouad F, Plence P, Bony C et al:: Immunosuppressive effect of mesenchymal stem cells favors tumor growth in allogeneic animals. Blood 102, 3837-3844 (2003).

3 Liu CH, Hwang SM: Cytokine interactions in mesenchymal stem cells from cord blood. Cytokine 32, 270-279 (2005).

4 Zacharek A, Chen J, Cui X et al.: Angiopoietin1/Tie2 and VEGF/Flk1 induced by MSCs treatment amplifies angiogenesis and vascular stabilization after stroke. J. Cereb. Blood Flow Metab. 27, 1684-1691 (2007).

5 Bruno S, Bussolati B, Grange C et al.: $\mathrm{CD}_{133^{+}}$renal progenitor cells contribute to tumor angiogenesis. Am. J. Pathol. 169, 2223-2235 (2006).

6 Levina V, Marrangoni AM, DeMarco R, Gorelik E, Lokshin AE: Drug-selected human lung cancer stem cells: cytokine network, tumorigenic and metastatic properties. PLoS ONE 3(8), 3077 (2008).

7 Lang SH, Frame FM, Collins AT: Prostate cancer stem cells. J. Pathol. 217(2), 299-306 (2009).

8 Taylor RA, Risbridger GP: Prostatic tumor stroma: a key player in cancer progression. Curr. Cancer Drug Targets 8(6), 490-497 (2008).

9 Djouad F, Bony C, Apparailly F et al.: Earlier onset of syngeneic tumors in the presence of mesenchymal stem cells. Transplantation 82, 1060-1066 (2006).

10 Houghton J, Stoicov C, Nomura S et al.: Gastric cancer originating from bone marrow-derived cells. Science 306, 1568-1571 (2004).

11 Miura M, Miura Y, Padilla-Nash HM et al.: Accumulated chromosomal instability in murine bone marrow mesenchymal stem cells leads to malignant transformation. Stem Cells 24, 1095-1103 (2006).

12 Klonish T, Wiecher E, Hombach-Klonish S: Cancer stem cell markers in common cancers-therapeutic implications. Trends Mol. Med. 14 (10), 450-460 (2008).
13 Ruiz i Altaba A: Therapeutic inhibition of Hedgehog-GLI signaling in cancer: epithelial, stromal, or stem cell targets? Cancer Cell 14(4), 281-283 (2008).

14 Li H, Han Y, Guo Q, Zhang M, Cao X: Cancer expanded myeloid derived suppressor cells induce anergy of NK cells through membrane bound TGF $\beta 1$. J. Immunol. 182, 240-249 (2009).

15 Scanlan MJ, Raj BK, Calvo B et al.: Molecular cloning of fibroblast activation protein alpha, a member of the serine protease family selectively expressed in stromal fibroblasts of epithelial cancers. Proc. Natl Acad. Sci. USA (12), 5657-5661 (1994).

16 Lazennec G, Jorgensen C: Adult multipotent stromal cells and cancer: risk or benefit? Stem Cells 26(6), 1387-1394 (2008).

17 Ohlsson LB, Varas L, Kjellman C et al.: Mesenchymal progenitor cell-mediated inhibition of tumor growth in vivo and in vitro in gelatin matrix. Exp. Mol. Pathol. 75, 248-255 (2003).

18 Nakamura K, Ito Y, Kawano Y et al.: Antitumor effect of genetically engineered mesenchymal stem cells in a rat glioma model. Gene Ther. 11, 1155-1164 (2004). 
19 Khakoo AY, Pati S, Anderson SA et al.: Human mesenchymal stem cells exert potent antitumorigenic effects in a model of Kaposi's sarcoma. J. Exp. Med. 203, 1235-1247 (2006).

20 Bierie B, Stover DG, Abel TW et al: Transforming growth factor $\beta$ regulates mammary carcinoma cell survival and interaction with the adjacent microenvironment. Cancer Res. 68(6), 1809-1819 (2008).

21 Karnoub AE, Dash AB, Vo AP et al.: Mesenchymal stem cells within tumor stroma promote breast cancer metastasis. Nature 449, 557-563 (2007).

22 Croitoru-Lamoury J, Lamoury FM, Zaunders JJ, Veas LA, Brew BJ: Human mesenchymal stem cells constitutively express chemokines and chemokine receptors that can be upregulated by cytokines, IFN- $\beta$, and Copaxone. J. Interferon Cytokine Res. 27(1), 53-64 (2007).

23 Corcoran KE, Trzaska KA, Fernandes $\mathrm{H}$ et al:: Mesenchymal stem cells in early entry of breast cancer into bone marrow. PLoS ONE 3(6), e2563 (2008).
24 Todaro M, Perez A, DiStefano A, Cammereri P: Colon cancer stem cells dictate tumor growth and resist cell death by production of IL4. Cell Stem Cell 1, 389-402 (2007).

25 Gunn WG, Conley A, Deininger L et al.: A crosstalk between myeloma cells and marrow stromal cells stimulates production of DKK1 and interleukin-6: a potential role in the development of lytic bone disease and tumor progression in multiple myeloma. Stem Cells 24, 986-991 (2006).

26 Fritz V, Jorgensen C: Mesenchymal stem cells: an emerging tool for cancer targeting and therapy. Curr. Stem Cell Res. Ther. 3(1), 32-42 (2008).

27 Li X, Lu Y, Huang W et al.: In vitro effect of adenovirus-mediated human $\gamma$-Interferon gene transfer into human mesenchymal stem cells for chronic myelogenous leukemia. Hematol. Oncol. 24, 151-158 (2006).

28 Chen XC, Wang R, Zhao X et al:: Prophylaxis against carcinogenesis in three kinds of unestablished tumor models via IL12-gene-engineered MSCs. Carcinogenesis 27, 2434-2441 (2006).
29 Xin H, Kanehira M, Mizuguchi $\mathrm{H}$ et al:: Targeted delivery of CX3CL1 to multiple lung tumors by mesenchymal stem cells. Stem Cells 25(7), 1618-1626 (2007).

30 Kanehira M, Xin H, Hoshino K et al: Targeted delivery of NK4 to multiple lung tumors by bone marrow-derived mesenchymal stem cells. Cancer Gene Ther. 14(11), 894-903 (2007).

31 Fritz V, Noël D, Bouquet C et al: : Antitumoral activity and osteogenic potential of mesenchymal stem cells expressing the urokinase-type plasminogen antagonist amino-terminal fragment in a murine model of osteolytic tumor. Stem Cells 26(11), 2981-2990 (2008).

\section{Website}

101 1st Conference on Mesenchymal Stromal Cells and Tumor Microenvironment: An Update. http://u844.free.fr/meeting.html 